Rabaska

Revue d'ethnologie de l'Amérique française

\title{
Autour de l’oeuvre de Marius Barbeau
}

\section{Jean-Pierre Pichette}

Volume 13, 2015

Présence de Marius Barbeau : l'invention du terrain en Amérique

française. Autour d'un legs centenaire (1914-2014)

URI : https://id.erudit.org/iderudit/1033743ar

DOI : https://doi.org/10.7202/1033743ar

Aller au sommaire du numéro

Éditeur(s)

Société québécoise d'ethnologie

ISSN

1703-7433 (imprimé)

1916-7350 (numérique)

Découvrir la revue

Citer ce document

Pichette, J.-P. (2015). Autour de l'oeuvre de Marius Barbeau. Rabaska, 13, 8-15.

https://doi.org/10.7202/1033743ar d'utilisation que vous pouvez consulter en ligne.

https://apropos.erudit.org/fr/usagers/politique-dutilisation/ 


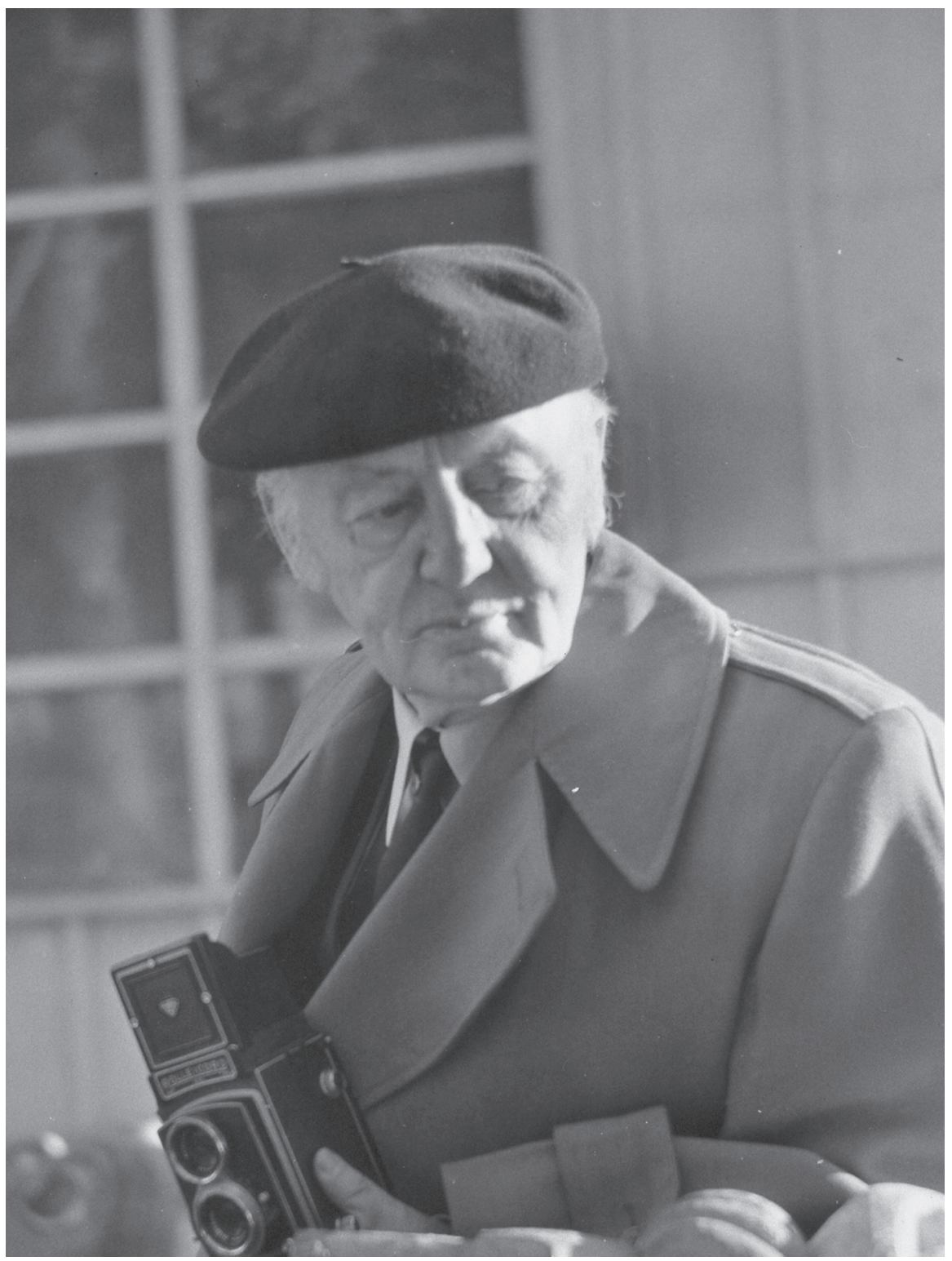

Marius Barbeau avec son appareil photo, vers 1960.

Source : Musée canadien de l'histoire, $n^{\circ}$ 2004-021. 


\section{Ouverture}

Autour de l'œuvre de Marius Barbeau

JeAn-Pierre Pichette

Société québécoise d'ethnologie

Professeur associé, Université Sainte-Anne

\section{Marius Barbeau}

Anthropologue et folkloriste, né à Sainte-Marie-de-Beauce, Québec, le 5 mars 1883, Frédéric-Charles-Joseph-Marius Barbeau est le fils aîné de Charles Barbeau, cultivateur et maquignon, grand connaisseur de traditions populaires, et de Virginie Morency, musicienne et ancienne novice chez les sœurs Grises, qui se chargea entièrement de l'éducation de son fils jusqu'à l'âge de douze ans. Ayant choisi de faire des études, le jeune Barbeau fit son cours classique au Collège de Sainte-Anne-de-la-Pocatière (1897-1903). Inscrit en droit à l'Université Laval (1903-1907), il obtint sa licence et fut admis au barreau ; mais la profession d'avocat ne l'attire pas et, à la faveur d'une bourse CecilRhodes, il se rend en Angleterre étudier le droit criminel au collège Oriel d'Oxford (1907-1910). Ce domaine ne lui convenant pas non plus, son directeur d'études, Reynold Marrett, l'oriente plutôt vers l'anthropologie ; il obtiendra en 1910 un diplôme en cette matière après avoir soumis sa thèse sur les Indiens du Pacifique ${ }^{1}$. Parallèlement à ses études à Oxford, Barbeau profite de ses vacances, trois mois par année, pour assister aux cours de l'École des hautes études à la Sorbonne et de l'École d'anthropologie à Paris. Il suit les leçons de Marcel Mauss (1873-1950) avec qui il se lie d'amitié. Au terme de ses études, il rentre au pays où, sur la recommandation de sir William Osler, un Canadien vivant à Oxford, il obtient un poste d'anthropologue à la Commission géologique du Canada (qui deviendra le Musée national du Canada en 1927) le $1^{\text {er }}$ janvier 1911. Il y fera carrière et résidera à Ottawa jusqu'à sa retraite en 1948 .

Scientifique bien en vue, il devint membre de l'American Folklore Society en 1911 et, à compter de 1916, coéditeur du Journal of American Folk-Lore, fondateur des sections française et anglaise de cette société au Canada en 1917, et même président en 1918. Élu à la Société royale du Canada en 1916,

1. Marius Barbeau, «The Totemic System of the North Western Indian Tribes of North America », thèse de baccalauréat en sciences présentée à 1'Université d'Oxford, 1910, 135 p. 
nommé président du Comité consultatif national pour la protection de la faune au Canada (1937), il fut aussi membre de l'Association canadienne des auteurs (1939), de l'Académie des sciences de Washington (1939) et de la Société des écrivains canadiens (1939), membre fondateur de l'Académie canadiennefrançaise (1945) et de la Société historique du Canada, et président fondateur de la Société canadienne de musique folklorique (1957). Orateur recherché, Marius Barbeau fut professeur invité dans plusieurs universités : d'abord conférencier aux cours d'été de l'Université Laval (1942), il enseigna la géographie humaine à l'Université d'Ottawa (1942) et donna des conférences en anthropologie et folklore à l'Université de Montréal (1945); mais, nommé professeur agrégé de Laval en 1945, il cessa sa collaboration avec les autres universités et il rédigea à l'intention de ses étudiants un précieux manuscrit intitulé « En quête de connaissances anthropologiques et folkloriques dans l'Amérique du Nord depuis $1911^{2} »$; il fut nommé professeur émérite en 1954.

En outre, plusieurs de ses livres lui ont mérité des prix littéraires : il obtint le prix David en 1925 pour son ouvrage Indian Days in the Canadian Rockies ${ }^{3}$; il le reçut une deuxième fois en 1929 pour The Downfall of Temlaham ${ }^{4}$ et une troisième fois en 1945 pour Saintes Artisanes I-Les brodeuses 5 . En 1946, l'Association canadienne-française pour l'avancement des sciences (ACFAs) lui remit la Médaille Léo-Parizeau. Puis on reconnut la valeur de son œuvre par plusieurs titres honorifiques : trois doctorats honoris causa (Université de Montréal, 1938 ; Laval, 1952 ; Oxford, 1953), compagnon honoraire du Collège Oriel d'Oxford (1941) et prix national en musique de l'Université de l'Alberta (1965). Enfin, il fut fait compagnon de l'Ordre du Canada (1967). Il est mort à Ottawa le 27 février 1969.

\section{Colloque international Organisation}

La Société québécoise d'ethnologie ( $\mathrm{SQE})$ et l'Université Sainte-Anne, par sa chaire de recherche CoFram, ont développé depuis une bonne décennie l'habitude d'œuvrer de concert à l'étude et à la mise en valeur du patrimoine ethnologique, des objectifs qu'elles possèdent en partage. La publication de

2. Marius Barbeau, "En quête de connaissances anthropologiques et folkloriques dans l'Amérique du Nord depuis 1911 » (Résumé d'un cours donné à la faculté des Lettres, mars-octobre 1945), Université Laval, Archives de folklore, 1945, 82 p.

3. Marius Barbeau, Indian Days in the Canadian Rockies, Toronto, The Macmillan Company of Canada, 1923, 208 p.

4. Marius Barbeau, The Downfall of Temlaham, Toronto, The Macmillan Company of Canada, 1928, XII-253 p. L'auteur publiera une version française remaniée de ce roman : Le Rêve de Kamalmouk, Montréal, Fides, « Nénuphar », 1948, 231 p.

5. Marius Barbeau, Saintes Artisanes I - Les brodeuses, Montréal, Éditions Fides, « Cahiers d'art Arca » II, [1944], 116 p. 
Rabaska, revue d'ethnologie de l'Amérique française, et la série, déjà longue, d'ateliers et d'échanges scientifiques tenus à Pointe-de-l'Église, à Sudbury, à Québec et à Brest particulièrement, en incarnent les principaux moments ${ }^{6}$. Les liens que ces entités ont aussi tissés avec le Centre de recherche bretonne et celtique (CRBC) de l'Université de Bretagne occidentale et divers corps de la francophonie nord-américaine et européenne, se sont récemment actualisés dans un nouveau partenariat. Conjointement avec la Société d'histoire de Charlevoix et le Centre de recherche sur l'histoire et le patrimoine de Charlevoix, ces institutions s'engageaient à explorer le « legs centenaire » de Marius Barbeau, sur les lieux mêmes d'un de ses terrains les plus fertiles. Ce colloque international, «Présence de Marius Barbeau ou l'invention du terrain en Amérique française », qui prend ainsi les airs d'une excursion intellectuelle guidée par nos collègues chercheurs du pays, s'inscrit pleinement parmi les célébrations entourant le trentième anniversaire de la Société d'histoire de Charlevoix, dont la mission de valorisation du patrimoine fait écho aux enquêtes du pionnier qui est au cœur de l'événement. De plus, les actes de cette manifestation paraissent en l'année du quarantième anniversaire de fondation de la Société québécoise d'ethnologie, un organisme national qui poursuit fidèlement sa mission de promouvoir l'ethnologie de la francophonie nord-américaine, et de favoriser la sauvegarde et la mise en valeur de son patrimoine ethnologique, tant sur le plan matériel qu'immatériel. Pour en diffuser les résultats, la convergence de ces divers facteurs ne pouvait désigner plus favorable véhicule que les pages mêmes de la revue Rabaska, organe de la Société québécoise d'ethnologie, qui les a réunis dans ce qui constitue son premier volume thématique.

\section{Problématique}

L'année 2014 marquait le centième anniversaire de la découverte de la littérature orale au Canada français à la suite du questionnement de Franz Boas, alors professeur à l'université Columbia. Leur rencontre à New-York à la fin décembre 1913 fut déterminante, car Barbeau sera le premier à s'intéresser scientifiquement au conte populaire des Français d'Amérique puis il relancera les recherches sur la chanson traditionnelle. Ce faisant, il fera déborder son affectation scientifique, l'ethnographie des sociétés amérindiennes, dans le champ du folklore et de la culture matérielle des Canadiens français qu'il imposera même au Musée national. Il a abondamment publié dans ces deux domaines - une soixantaine de livres et des centaines d'articles -, tant en

6. Cf. les actes de ces colloques dans la revue Port Acadie : La Résistance des marges (2007; $\left.\mathrm{n}^{\mathrm{os}} 13-14-15,2008-2009\right)$, Éditer des contes de tradition orale. Pour qui ? Comment? (2008; ${ }^{\circ \mathrm{os}} 16-17$, 2009-2010) et L'Apport des prêtres et des religieux au patrimoine des minorités (2011; $\mathrm{n}^{\text {os }} 24-25-26$, 2013-2014). Ils sont aussi disponibles en ligne par le portail Érudit à l'adresse www.erudit.org/revue/pa. 
français qu'en anglais, au point qu'on le considère comme le pionnier de ces disciplines scientifiques ${ }^{7}$. Barbeau fut vraisemblablement celui de tous les folkloristes et ethnologues canadiens qui a consacré le plus de temps au travail de terrain, parfois jusqu'à cinq mois par année. Sa collection de littérature orale franco-canadienne compte à elle seule environ 13000 chansons, dont 6000 avec la mélodie recueillie au phonographe, et plus de 300 contes consignés à la sténographie ${ }^{8}$. Aussi, avons-nous souhaité examiner cet aspect particulier de l'œuvre de Barbeau par un colloque international, lequel, grâce à nos partenaires, se tiendrait précisément dans la région de Charlevoix qui fut l'un de ses terrains privilégiés. Une trentaine de chercheurs du Québec, du Canada, des États-Unis et de la France répondirent à l'appel à communiquer, lancé et relayé par l'entremise de nos réseaux de chercheurs, et se réunirent ainsi à Saint-Irénée à l'automne 2014 afin d'alimenter l'un ou l'autre des axes de réflexion qui structuraient la thématique générale : «Présence de Marius Barbeau - L'invention du terrain en Amérique française - Autour d'un legs centenaire (1914-2014)».

\section{Les actes du colloque}

Ce compte rendu reprend l'essentiel de la matière du colloque et dispose les interventions en deux parties : Enquête, terrain et territoire et Promotion et mise en valeur du patrimoine.

\section{Première partie - Enquête, terrain et territoire}

L'enquête orale, la forme de documentation par laquelle Marius Barbeau imprima un virage scientifique à la discipline, représente un exercice indispensable. Ses recherches commandent un séjour sur le terrain qui, seul, permet d'appréhender les faits et dits populaires sans intermédiaire, directement auprès des témoins qui peuvent les transmettre et renseigner le chercheur. Si ce dernier sait s'adapter à son terrain, il doit pratiquer « l'observation parfaite ", comme Barbeau le professait à ses étudiants de l'Université Laval en 1945 ; il posait néanmoins une condition pour ce faire : « Des connaissances préparatoires des sujets à découvrir et à étudier sont nécessaires. Le chercheur, au cours de sa carrière, doit souvent se préparer à l'observation parfaite d'un domaine nouveau pour lui. [...] Le choix de l'objet des recherches et de l'endroit où les poursuivre, constitue le premier pas. Le flair et les renseigne-

7. Clarisse Cardin, «Bio-bibliographie de Marius Barbeau », dans Les Archives de folklore, [Montréal], Éditions Fides, « Publications de l’Université Laval », vol. 2 « Hommage à Marius Barbeau », 1947, p. [17]-96.

8. Faute d'un décompte officiel, nous reprenons l'estimé qu'en donnait le principal intéressé dans «Les Mémoires de Marius Barbeau », enregistrés et transcrits par Carmen Roy, Ottawa, Musée canadien des civilisations, 1958, p. 341. 
ments peuvent aider dans ce choix, ainsi que les circonstances. ${ }^{9} \gg$ Ainsi, avant d'y mettre les pieds, Barbeau avait apprivoisé son sujet, s'était familiarisé au préalable avec le milieu, son passé et ses particularités, avait établi des contacts, d'autant qu'il désirait documenter le domaine de l'oralité, un champ méconnu, voire méprisé par les intellectuels de son temps. La cartographie de ses lieux d'enquête comme l'examen de ses écrits sur le « pays des gourganes » et le « Saguenay légendaire » exposent une facette de sa méthode et montrent le regard qu'il posait sur le patrimoine oral recueilli en Charlevoix et les détenteurs de la tradition.

La valeur de son bilan apparaît dès qu'on s'interroge sur sa contribution dans les genres de la littérature orale qu'il a privilégiés et dans les autres domaines qu'il a touchés. Comment la mesurer? Que lui a apporté le terrain, soit l'enquête orale directe, comme source de savoir? Que valent aujourd'hui ses travaux?

Barbeau et l'oralité. Si, par la pratique du terrain, il a pu engranger des pans importants de l'oralité des Français d'Amérique, spécialement en chanson et en conte traditionnels dont il est le découvreur incontestable, il a diffusé les fruits de ses explorations de multiples façons. Leur parution dans des revues savantes reconnues, comme le Journal of American Folklore, marque d'une pierre blanche cet ancrage scientifique au Canada, tant ses écrits suscitent encore l'admiration des spécialistes par le foisonnement de ses investigations et la qualité de leur traitement qui reste fidèle au matériau oral. Pour la chanson, son Romancero du Canada demeure le livre phare qui a fasciné et inspiré les chercheurs qui ont suivi ; Le rossignol y chante et les autres tomes $\mathrm{du}$ « Répertoire de la chanson folklorique française au Canada» auront plus tard des répercussions inattendues sur de jeunes enquêteurs. Néanmoins, soucieux de transmettre cette littérature populaire aux nouvelles générations, Barbeau s'est fait « passeur », médiateur culturel, en adaptant régulièrement ses écrits à des publics variés, notamment dans des ouvrages " pour lecture générale », dont certains destinés à la jeunesse ou aux touristes, mais aussi en animant des festivals du terroir pour un auditoire local ou étranger. Au milieu des années 1920, il étendra ses investigations à l'art religieux, portant son attention sur le patrimoine des églises et des communautés religieuses, et leurs artisans, puis à l'art populaire, toujours en documentant les techniques populaires et en militant pour leur sauvegarde.

\section{Deuxième partie - Promotion et mise en valeur du patrimoine}

Barbeau et ses alliés. Marius Barbeau a su communiquer sa passion à de nom-

9. Marius Barbeau, « En quête de connaissances anthropologiques et folkloriques [...]», op. cit., p. 45 . 
breux collaborateurs, sollicitant la mémoire de son entourage pour enrichir ses écrits ou s'alliant en région à des autorités réputées dont la contribution s'avérera capitale. On entrouvre ici le dossier des relations épistolaires soutenues qu'il a cultivées avec plusieurs correspondants dans le champ de l'oralité principalement : l'archiviste montréalais Édouard-Zotique Massicotte, l'historien saguenéen Victor Tremblay, le collectionneur franco-américain Adélard Lambert et le journaliste acadien Joseph-Thomas LeBlanc. En scrutant les archives du Musée canadien de l'histoire, qui conservent les traces abondantes du chercheur, on saisit toutefois son engagement évident dans de nombreuses autres sphères : par exemple, son apport remarquable à la muséologie -à des titres divers : collectionneur, conservateur, commissaire, auteur consulté au Canada et à l'étranger -, ou à la connaissance des techniques artisanales que les expertises scientifiques actuelles de ses notes ethnographiques permettent de préciser, sans compter ses initiatives de collecte et diffusion de bien d'autres aspects de la culture traditionnelle.

Ethnologie et université. L'inscription en 1944 de la tradition dans le programme universitaire de Laval, axée d'abord sur l'oralité puisqu'elle émergeait de la littérature, couronna la carrière de Barbeau. Si, en France, on fait remonter l'avènement de l'ethnologie à 1937, ses contemporains - Arnold Van Gennep, Patrice Coirault, Paul Delarue - menèrent leurs travaux isolément, en marge de l'institution, le terme « folklore » connotant l'amateurisme et le régionalisme. L'exemple breton montre comment l'ethnologie parvint à prendre sa place grâce à la caution de chercheurs du Centre national de la recherche scientifique (CNRS), Jean-Michel Guilcher en tête, qui mirent sur pied dans les années 1970 un enseignement lié au Centre de recherche bretonne et celtique avant de créer un département autonome qui en décline depuis 1990 toutes les formations, de la licence au doctorat. En cette année du soixante-dixième anniversaire du programme initial lavalois et en dépit des résultats obtenus, on a évoqué la suspension du volet fondateur de l' " orature » à travers les mutations et les innovations de cet enseignement, mais aussi la précarité même des programmes d'ethnologie en milieu universitaire à l'heure de l'« austérité » des finances publiques.

\section{Épilogue. - Un exemple pour demain}

Néanmoins, le corpus patrimonial qu'a constitué et diffusé ce précurseur durant plus d'un demi-siècle a toujours sa pertinence. Sa quête des traditions par l'enquête orale auprès des Français d'Amérique connaît des suites durables, car elle a renouvelé les connaissances en patrimoine, tant dans le 
domaine de l'oralité que dans les autres composantes de la tradition. Les chercheurs d'aujourd'hui témoignent de sa validité, tantôt dans de grands projets d'inventaire, tantôt dans des domaines ciblés ou dans le cadre d'applications journalistiques, le terrain demeurant l'outil primordial de la démarche ethnologique auprès du témoin qui a vu et qui a vécu les faits à l'étude, avec le concours des nouvelles technologies qui facilitent le travail. 